\title{
Unrequested information from routine diagnostic chest CT predicts future cardiovascular events
}

\author{
Peter C. Jacobs • Martijn J. Gondrie • Willem P. Mali • \\ Ayke L. Oen • Mathias Prokop • Diederick E. Grobbee • \\ Yolanda van der Graaf
}

Received: 24 August 2010 /Revised: 22 November 2010 / Accepted: 23 December 2010 /Published online: 21 May 2011

(C) The Author(s) 2011. This article is published with open access at Springerlink.com

\begin{abstract}
Objectives An increase in the number of CT investigations will likely result in a an increase in unrequested information. Clinical relevance of these findings is unknown. This is the first follow-up study to investigate the prognostic relevance of subclinical coronary (CAC) and aortic calcification (TAC) as contained in routine diagnostic chest CT in a clinical care population.

Methods The follow-up of 10,410 subjects ( $>40$ years) from a multicentre, clinical care-based cohort of patients included 240 fatal to 275 non-fatal cardiovascular disease (CVD) events (mean follow-up 17.8 months). Patients with a history of CVD were excluded. Coronary (0-12) and aortic calcification $(0-8)$ were semi-quantitatively scored.
\end{abstract}

P. C. Jacobs $(\bowtie) \cdot$ M. J. Gondrie • D. E. Grobbee

Y. van der Graaf

Julius Center for Health Sciences and Primary Care,

University Medical Center Utrecht,

Universiteitsweg 100, P.O. Box 85500, 3508 GA Utrecht,

The Netherlands

e-mail: p.c.a.jacobs@umcutrecht.nl

P. C. Jacobs $\cdot$ W. P. Mali

Department of Radiology, University Medical Center Utrecht, Heidelberglaan 100,

3584 CX Utrecht, The Netherlands

\section{A. L. Oen}

Department of Radiology, Elkerliek Medical Center Helmond,

Wesselmanlaan 25,

5707 HA Helmond, The Netherlands

\section{Prokop}

Department of Radiology,

Radboud University Nijmegen Medical Center,

Geert Grooteplein Zuid 10,

6525 GA Nijmegen, The Netherlands
We used Cox proportional-hazard models to compute hazard ratios to predict CVD events.

Results CAC and TAC were significantly and independently predictive of CVD events. Compared with subjects with no calcium, the adjusted risk of a CVD event was 3.7 times higher (95\% CI, 2.7-5.2) among patients with severe coronary calcification (CAC score $\geq 6$ ) and 2.7 times higher (95\% CI, 2.0-3.7) among patients with severe aortic calcification (TAC score $\geq 5$ ).

Conclusions Subclinical vascular calcification on CT is a strong predictor of incident CVD events in a routine clinical care population.

Keywords Coronary artery calcification $\cdot$ Computed tomography $\cdot$ Cardiovascular disease $\cdot$ Stroke $\cdot$ Prevention

\section{Introduction}

Over the last decades, advances in diagnostic imaging techniques have been impressive. At the same time, this success has resulted in an increase in unrequested information during the diagnostic work-up of patients. Unrequested information can be defined as imaging abnormalities of potential clinical relevance that are unexpectedly discovered and unrelated to the clinical indication. Although unrequested information occurs across different imaging techniques and anatomical regions, computed tomography (CT) of the chest provides numerous exemplary cases of unrequested information: vascular calcifications, pulmonary emphysema, pulmonary nodules, or skeletal osteoporosis. Due to lack of follow-up, the clinical importance of unexpected abnormalities is largely unknown. Performing follow-up studies is the only way to determine which imaging abnormalities have or do not have clinical 
relevance. Alternatively, unrequested information could also contain valuable prognostic information on a variety of prevalent diseases in an ageing western population providing a new means of identifying patients that could profit from primary prevention efforts.

Non-invasive assessment of coronary artery calcium (CAC) by various $\mathrm{CT}$ techniques has been widely used as a prognostic marker of cardiovascular disease (CVD) in large prospective screening studies [1-5]. Vascular calcifications have a high prevalence in the normal population [6] and will thus be detected frequently when reading diagnostic $\mathrm{CT}$ in a clinical care population. To date, no follow-up studies have been performed to investigate whether vascular calcifications - as a prevalent example of unrequested information-can be used as prognostic marker in a population receiving routine clinical care.

In this study we examined whether subclinical coronary and/or aortic calcification detected unexpectedly by routine diagnostic chest $\mathrm{CT}$ is associated with future CVD events.

\section{Methods}

\section{Population}

All subjects are participants of the PROVIDI study, a multicentre retrospective cohort study among persons 40 years of age and older who underwent chest $\mathrm{CT}$ in one of 8 participating hospitals between January 2002 and December 2005. In patients with more than one chest CT examination during follow-up, only the first CT scan was used for analysis. The original cohort consisted of 23,443 patients. Based on CT reports, a research physician excluded all patients $(n=9077)$ with a poor prognosis defined as (1) diagnosis of primary lung cancer (including mesothelioma), or (2) diagnosis of distant metastatic disease from other types of cancer (not including haematological malignancies). This exclusion criterion was applied as it is highly unlikely that in patients with a poor prognosis detection of subclinical disease markers will alter clinical decision making. Similarly, subjects with suspected cardiovascular disease or patients known to have symptomatic cardiovascular disease were excluded $(n=2303)$. Starting May 2008, CT data of all eligible patients were transferred to the primary study site to be evaluated for the presence of unrequested information.

The institutional review boards of all participating centres approved this study, and written informed consent was waived because of the retrospective design of this study; a privacy protocol was implemented.

After exclusion, a total of 12,063 patients were eligible for this study. All 1653 patients from one randomly chosen study centre were additionally excluded to be used as an external validation sample for future research. Chest CT examinations were thus available for 10,410 patients. A flow-chart detailing the inclusion and exclusion steps for this research project is provided as Appendix to this study.

Study design

We used a case-cohort design in which a representative subcohort $(n=1285)$ is randomly sampled from the baseline cohort $(n=10,410)$ at the start of the study. This random sample should be $>10 \%$ of the size of the baseline cohort [7]. All participants in the baseline cohort are followed-up for the outcome-of-interest. Incident cases plus the random subcohort define the actual population under study. In the data analysis, all members of the subcohort are weighted by the inverse sampling fraction to reach valid estimates for the full cohort. This design provides an efficient way to avoid scoring CT characteristics for all 10,410 subjects of the baseline cohort, but instead only in incident cases and a representative baseline sample.

\section{Chest CT protocols}

To mimic day-to-day clinical practice and to increase the generalisability of our results, we chose to include CT from a wide spectrum of different $\mathrm{CT}$ machines to imaging protocols. All CT were obtained with dual, 4-, 8-, 16-, 40-, or 64-slice systems from different vendors. The field-ofview (FOV) of all eligible chest CT protocols had to include the heart and the full length of the thoracic aorta. Slice thickness varied according to the $\mathrm{CT}$ indication and corresponding protocol: e.g. $3 \mathrm{~mm}$ for $\mathrm{CT}$ pulmonary embolism, $5 \mathrm{~mm}$ for standard chest $\mathrm{CT}$ and $10 \mathrm{~mm}$ for highresolution $\mathrm{CT}$ of the lung parenchyma.- $\mathrm{CT}$ examinations with and without intravenous contrast enhancement were used. One research physician abstracted and classified the CT indication on the basis of information from CT reports; examples include suspected pulmonary disease, cardiovascular disease, traumatic injury, or mediastinal disease.

\section{Scoring of CT characteristics}

Computed tomography data sets were scored with regard to coronary and aortic calcification by three readers: one board certified radiologist with 10 years of experience and two research physicians with 2 and 3 years of experience in reading chest $\mathrm{CT}$. All readers were blinded for patient characteristics and outcome status. CT images were stored in DICOM format and read using a commercially available software program (Dicomworks, version 1.3.5). All CT were analysed in the axial plane; no other reformatted planes or thin slice reconstructions were included for analysis. Readings were performed using a standardised 
score form including information on the type of CT protocol (section thickness, tube load (mAs) and voltage $(\mathrm{kVp})$, and the use of contrast agent) and image quality (defined as good, adequate or poor).

The extent of vascular calcification was scored using simple visual grading (0-3) as previously described [8]. Table 1 provides exact definitions. Briefly, the four main coronary artery branches were scored separately $(0-3)$ before being summed into a single score for the coronary artery tree $(0-12)$. Calcification of the thoracic aorta was scored in three locations: calcification in the ascending $(0-3)$ and descending $(0-3)$ aorta and calcification in the supra-aortic arteries branching of the aortic arch (0-2), which were summed into a single score for the thoracic aorta (0-8). The lower cut-off level for the descending aorta was defined as the mid-level of the 11th thoracic vertebra. Figure 1 provides a visual example of different grades of CAC.

Use of the score form was trained under the supervision of an experienced board certified chest radiologist using a training set of 50 randomly selected patients. Reproducibility of visual grading was evaluated. Briefly, weighted kappa for inter -and intra-observer variability of aortic wall abnormalities (including calcifications) were 0.72 and 0.88 , for calcifications of the supra-aortic arteries 0.89 and 0.96 , and for calcifications in the coronary arteries 0.77 and 0.91 , respectively.

\section{Follow-up}

We recorded incident fatal and non-fatal CVD events for a mean of 17.8 months. End-point status was obtained through linkage of patients with the National Death Registry and the National Registry of Hospital Discharge Diagnoses from January 2002 to December 2005. Database linkage was performed with a validated probabilistic method [9-12]. In these databases, cause of death and the occasion of hospitalisation are coded according to the International Classification of Diseases, 9th and 10th revision (ICD-9, ICD-10) [13]. Correct designation of causes of death has been established with in a comparison study with patient medical records [14]. The incidence of CVD events in the full cohort of 10,410 participants was $n=515$.

A trained research physician abstracted all valid end points from these databases on the basis of ICD codes. Fatal and non-fatal CVD events were defined by ICD-9 codes as coronary heart disease (CHD) (codes 410-414), heart failure (code 428), peripheral arterial disease (PAOD) (codes 440, 443-444), aortic aneurysm (code 441), cerebrovascular disease (codes 430-438), or non-rheumatic valvular disease (code 424). All other codes included in the ICD-9 paragraph on diseases of the circulatory system were not considered as valid end points for this study. In the case of multiple valid end points in the same patient, cause of death prevailed over hospital admissions or else the first hospital discharge diagnosis was used. Secondary analyses were performed for a selection of cases from the primary end point: all fatal and non-fatal coronary events (codes 410-414) and all fatal and non-fatal non-cardiac events (PAOD, codes 440, 443-444; aortic aneurysm, code 441; and cerebrovascular events, codes 430-438).

\section{Data analysis}

Differences in risk factors and $\mathrm{CT}$ protocols between subjects from the subcohort to various case-groups were tested with Chi-squared tests (discrete variables) and oneway ANOVA (continuous variables). Next, we calculated annualised event rates for all three end points stratified according to coronary and aortic calcium scores.

We used Cox proportional-hazards regression to estimate hazard ratios for any CVD event, coronary events, and any non-cardiac event according to calcium scores. Sum scores for visually graded coronary and aortic calcifications were analysed as categorical and as continuous variables (per SD). Categories of the aortic calcification sum score (0-8)

Table 1 Definitions used for visual grading of calcified plaques on routine diagnostic chest CT

\begin{tabular}{|c|c|c|c|c|}
\hline \multirow[t]{2}{*}{ Finding (potential range) } & \multicolumn{4}{|l|}{ Grades } \\
\hline & 0 & 1 & 2 & 3 \\
\hline $\begin{array}{l}\text { Aortic wall calcification }(0-3) \\
\text { (score for ascending and } \\
\text { descending aorta separately) }\end{array}$ & absent & $\leq 3$ foci & $\begin{array}{l}\text { 4-5 foci or } 1 \text { calcification } \\
\text { extending over } \geq 3 \text { slices }^{\mathrm{a}}\end{array}$ & $\begin{array}{l}>5 \text { foci or } 1 \text { calcification } \\
\text { extending over } \geq 3 \text { slice }\end{array}$ \\
\hline Supra-aortic artery calcification $(0-2)$ & absent & $\begin{array}{l}\text { calcifications in } 1 \\
\text { supra-aortic artery }\end{array}$ & $\begin{array}{l}\text { calcifications in }>1 \text { supra-aortic } \\
\text { arteries }\end{array}$ & \\
\hline $\begin{array}{l}\text { Coronary calcification }(0-3) \text { (score per } \\
\text { main branch: LM,LAD,LCX, RCA) }\end{array}$ & absent & $1-2$ foci & $\begin{array}{l}>2 \text { foci or } 1 \text { calcification } \\
\quad \text { extending over } \geq 2 \text { slices }\end{array}$ & $\begin{array}{l}\text { calcified arteries covering a } \\
\text { large segment of a coronary } \\
\text { branch }\end{array}$ \\
\hline
\end{tabular}

${ }^{\text {a }}$ Values are based on standard chest CT protocols with $5 \mathrm{~mm}$ section thickness 
Fig. 1 Axial 16-slice CT images of different grades of coronary artery calcification in the left anterior descending (LAD) branch (arrows demonstrate the example area): a grade 1 ( 2 focal calcifications); b grade 2 (1 large calcification); c grade 3 (extensive calcification covering a large segment of the LAD branch)

were chosen as: no calcification (reference category), mild calcification (sum score 1-2), moderate calcification (sum score 3-4) and severe calcification (sum score 5 and higher). In the case of coronary calcifications $(0-12)$, category cut-offs were chosen as: no calcification (reference category), mild calcification (sum score 1-2), moderate calcification (sum score 3-5) and severe calcification (sum score 5 and higher).

After estimating the hazard ratios for all non-cardiac events according to the aortic sum score, we performed additional regression analyses for all cases of stroke, looking at calcifications in each anatomically distinct part of the thoracic aorta (ascending, descending, arch). Finally, we investigated the multivariate associations when coronary and aortic sum scores were modelled simultaneously.

Apart from crude associations, all models were adjusted for age, sex, indication for CT, image quality to type of medical centre in which the CT was performed (tertiary/ secondary). We also tested the interaction of aortic and coronary sum scores with section thickness (continuous) and the use of contrast agent (yes/no) by entering interaction terms into the model. We had missing values for CT characteristics in $<3 \%$. We used regression methods implemented in SPSS software (SPSS 14.0, Chicago, IL, USA) to impute missing values. All other analyses were performed with R software, version 6.2.

\section{Results}

A total of 116 subjects ( 39 of 515 cases (7.6\%), and 77 of 1285 subjects from the subcohort $(6.0 \%)$ ) were excluded from analyses because CT images could not be retrieved from CT databases. A total of 515 subjects had a CVD event during follow-up: fatal CVD events occurred in 240 patients, and 275 patients had a non-fatal event. Table 2 summarises the baseline characteristics of the subcohort and the various case-groups.

Table 3 shows a gradual increase in annualised event rate for any CVD event with increasing CAC and TAC score categories.

Subjects with coronary and non-cardiac events were older than people in the subcohort, but only subjects with coronary events were more often male.

The prevalence of CAC (sum score $>0$ ) was $67 \%$ for people in the subcohort compared with $88 \%$ in subjects with CVD events, $90 \%$ for coronary events, and $85 \%$ for non-cardiac events $(P<0.001$ for all three groups). The corresponding figures for TAC $>0$ were $61 \%$ (subcohort), $82 \%, 80 \%$ and $86 \%$, respectively $(P<0.001$ for all three groups).
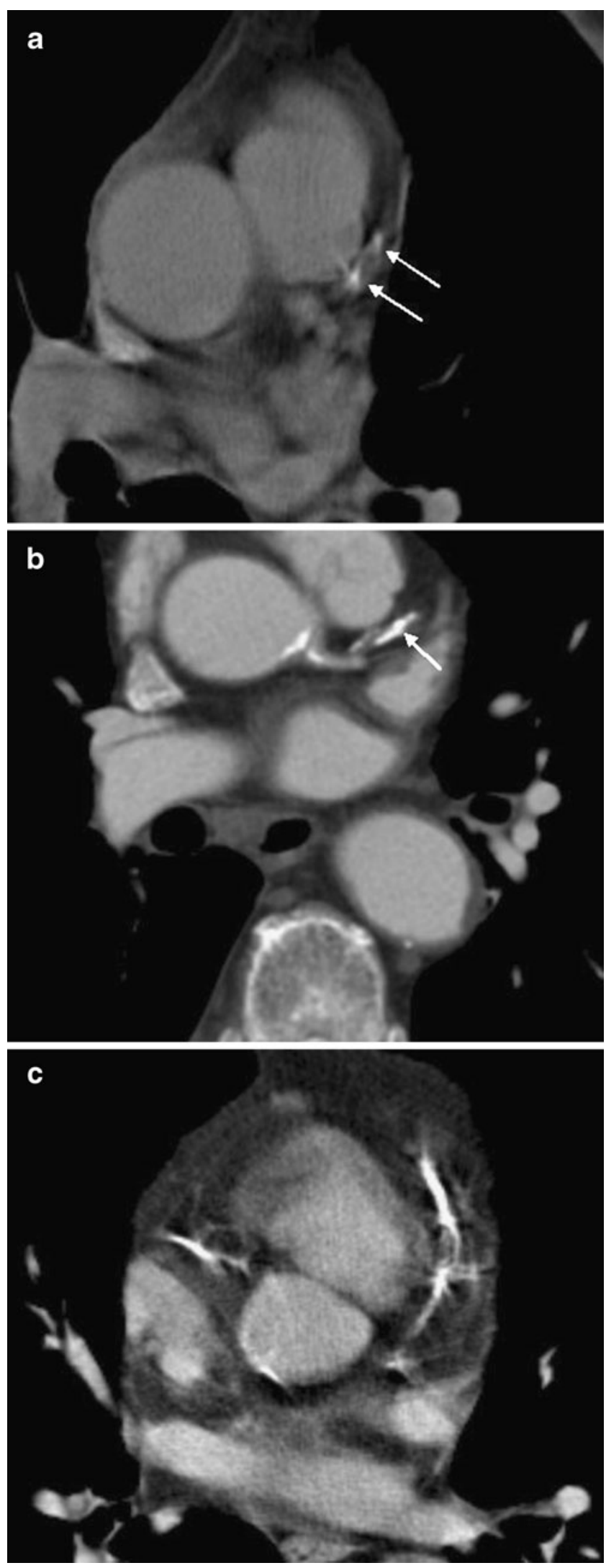

Table 4 shows that the risk of any CVD event increases with an increase in category of TAC and CAC scores after 
Table 2 Baseline characteristics

\begin{tabular}{|c|c|c|c|c|c|}
\hline & Variable & $\begin{array}{l}\text { Subcohort } \\
(N=1208)\end{array}$ & $\begin{array}{l}\text { Any CVD event } \\
(N=515)\end{array}$ & $\begin{array}{l}\text { Coronary event } \\
(N=310)\end{array}$ & $\begin{array}{l}\text { Non-cardiac event } \\
(N=128)\end{array}$ \\
\hline & Age (years) & $61.5 \pm 11.9$ & $67.9 \pm 11.3$ & $68.1 \pm 11.0$ & $67.8 \pm 11.3$ \\
\hline & Male sex $(\%)$ & 58 & 65 & 68 & 58 \\
\hline & \multicolumn{5}{|l|}{ CT indication $(\%)$} \\
\hline & Pulmonary disease & 37 & 47 & 50 & 37 \\
\hline & Haematological malignancy & 11 & 6 & 6 & 5 \\
\hline & Mediastinal disease & 11 & 8 & 10 & 8 \\
\hline & Ruled-out pulmonary malignancy & 24 & 21 & 18 & 27 \\
\hline & Pulmonary embolism & 6 & 8 & 6 & 12 \\
\hline & Other & 11 & 10 & 10 & 11 \\
\hline & \multicolumn{5}{|l|}{ Image quality (\%) } \\
\hline & Good & 72 & 74 & 76 & 69 \\
\hline & Adequate & 24 & 24 & 23 & 27 \\
\hline & Poor & 3 & 2 & 1 & 4 \\
\hline & Tertiary medical centre $(\%)$ & 77 & 77 & 78 & 73 \\
\hline & Use of contrast agent $(\%)$ & 68 & 67 & 66 & 70 \\
\hline & \multicolumn{5}{|l|}{ Section thickness (mm) } \\
\hline & $1-3 \mathrm{~mm}$ & 43 & 41 & 43 & 36 \\
\hline & $4-6 \mathrm{~mm}$ & 39 & 38 & 38 & 37 \\
\hline & $>6 \mathrm{~mm}$ & 18 & 21 & 19 & 27 \\
\hline & \multicolumn{5}{|l|}{ Thoracic aortic calcium (sum score) } \\
\hline & None $(0)$ & 39 & 18 & 20 & 14 \\
\hline & Mild (1-2) & 30 & 25 & 23 & 29 \\
\hline & Moderate (3-4) & 19 & 33 & 33 & 35 \\
\hline & Severe $(\geq 5)$ & 12 & 24 & 24 & 22 \\
\hline & \multicolumn{5}{|l|}{ Coronary artery calcium (sum score) } \\
\hline & None $(0)$ & 33 & 12 & 10 & 15 \\
\hline & Mild (1-2) & 24 & 22 & 20 & 25 \\
\hline & Moderate (3-5) & 25 & 29 & 28 & 27 \\
\hline Values are means \pm SD or & Severe $(\geq 6)$ & 18 & 37 & 42 & 32 \\
\hline
\end{tabular}

Values are means $\pm \mathrm{SD}$ or proportions

adjustment for age, sex, CT indication, CT image quality and type of medical centre. A statistically significant increase in hazard ratio occurs already in the case of mild calcification (TAC and CAC scores $=1-2$ ). The risk of any CVD event was increased by a factor of 2.7 (95\% CI, 2.0-3.7) and 3.7

Table 3 Annualised event rates for any CVD event $(n=515)$ according to coronary artery calcium score and thoracic aorta calcium score categories

Annualised event rates

\begin{tabular}{llcl}
\hline TAC risk category & \multicolumn{3}{c}{ CAC risk category } \\
\hline None (0) & $1.0(95)$ & None (0) & $0.7(62)$ \\
Mild (1-2) & $1.9(127)$ & Mild (1-2) & $2.2(111)$ \\
Moderate (3-4) & $5.0(170)$ & Moderate (3-5) & $3.1(150)$ \\
Severe $(\geq 5)$ & $5.6(123)$ & Severe $(\geq 6)$ & $5.9(192)$ \\
\hline
\end{tabular}

Data are percentages. Data in parentheses are numbers of patients. Median follow-up per case-group: 17.8 months for total CVD events
(95\% CI, 2.7-5.2), respectively, among patients with severe TAC or CAC (TAC score $\geq 5$; CAC score $\geq 6$ ) compared with those without any calcium. In order to compare the strength of the associations between the two calcium measures, Table 4 also shows the increase in risk of any CVD event associated with CAC and TAC as continuous measures (per $1 \mathrm{SD}$ increase in the TAC and CAC sum scores). After adjustment, an increase of $1 \mathrm{SD}$ in TAC and CAC sum scores resulted in a $46 \%$ and $41 \%$ increase in the risk of any CVD event.

Looking at coronary and non-cardiac events separately (Table 4, lower half), we could demonstrate a more differentiated pattern in the associations with TAC and CAC scores. Risk of coronary events increased 5-fold among subjects with severe CAC (CAC score $\geq 6$ ) compared with those without any coronary calcium, whereas having severe TAC (TAC score $\geq 5$ ) resulted in an increase in risk by a factor of 2.4. Conversely, risk of non-cardiac events (stroke, aortic aneurysm/dissection, PAOD) increased by a factor of 3.3 for subjects with severe TAC compared with patients without any aortic calcium, 
Table 4 Risk of any CVD event, coronary events, and non-cardiac events associated with increasing coronary artery calcium score and thoracic aorta calcium score in clinical care patients undergoing routine diagnostic chest $\mathrm{CT}$

\begin{tabular}{|c|c|c|c|c|c|}
\hline \multicolumn{3}{|l|}{ End points } & \multicolumn{3}{|c|}{ Hazard ratios $(95 \% \mathrm{CI})$} \\
\hline & Crude & Full model $^{\mathrm{a}}$ & & Crude & Full model ${ }^{\mathrm{a}}$ \\
\hline \multicolumn{6}{|c|}{ Any CVD event $(n=515)$} \\
\hline TAC categories & & & CAC categories & & \\
\hline None $(0)$ & 1.0 & 1.0 & None $(0)$ & 1.0 & 1.0 \\
\hline Mild (1-2) & $1.8(1.4-2.4)$ & $1.4(1.0-1.8)$ & Mild (1-2) & $2.8(2.0-3.8)$ & $2.2(1.6-3.0)$ \\
\hline Moderate (3-4) & $4.4(3.4-5.6)$ & $2.6(1.9-3.4)$ & Moderate (3-5) & $3.8(2.9-5.2)$ & $2.5(1.8-3.4)$ \\
\hline Severe $(\geq 5)$ & $4.9(3.8-6.4)$ & $2.7(2.0-3.7)$ & Severe $(\geq 6)$ & $6.9(5.2-9.2)$ & $3.7(2.7-5.2)$ \\
\hline Continuous $^{\mathrm{b}}$ & $1.76(1.64-1.90)$ & $1.46(1.33-1.60)$ & Continuous $^{\mathrm{b}}$ & $1.73(1.61-1.85)$ & $1.41(1.30-1.54)$ \\
\hline \multicolumn{6}{|c|}{ Coronary events $(n=310)$} \\
\hline TAC categories & & & CAC categories & & \\
\hline None $(0)$ & 1.0 & 1.0 & None $(0)$ & 1.0 & 1.0 \\
\hline Mild (1-2) & $1.5(1.1-2.2)$ & $1.2(0.8-1.7)$ & Mild (1-2) & $3.0(1.9-4.6)$ & $2.4(1.5-3.7)$ \\
\hline Moderate (3-4) & $4.0(3.0-5.5)$ & $2.3(1.6-3.3)$ & Moderate (3-5) & $4.3(2.9-6.4)$ & $2.9(1.9-4.4)$ \\
\hline Severe $(\geq 5)$ & $4.6(3.3-6.4)$ & $2.4(1.6-3.5)$ & Severe $(\geq 6)$ & $9.1(6.2-13.4)$ & $5.0(3.2-7.7)$ \\
\hline Continuous $^{\mathrm{b}}$ & $1.76(1.60-1.94)$ & $1.43(1.27-1.60)$ & Continuous ${ }^{\mathrm{b}}$ & $1.87(1.71-2.05)$ & $1.54(1.38-1.72)$ \\
\hline \multicolumn{6}{|c|}{ Non-cardiac events $(n=128)^{\mathrm{c}}$} \\
\hline TAC categories & & & CAC categories & & \\
\hline None $(0)$ & 1.0 & 1.0 & None $(0)$ & 1.0 & 1.0 \\
\hline Mild (1-2) & $2.6(1.5-4.5)$ & $1.9(1.1-3.4)$ & Mild (1-2) & $2.5(1.4-4.3)$ & $1.8(1.0-3.3)$ \\
\hline Moderate (3-4) & $5.6(3.3-9.7)$ & $3.5(1.9-6.4)$ & Moderate (3-5) & $2.7(1.6-4.7)$ & $1.7(0.9-3.0)$ \\
\hline Severe $(\geq 5)$ & $5.5(3.1-9.8)$ & $3.3(1.7-6.4)$ & Severe $(\geq 6)$ & $4.5(2.6-7.6)$ & $2.3(1.3-4.3)$ \\
\hline Continuous ${ }^{\mathrm{b}}$ & $1.71(1.47-1.98)$ & $1.45(1.21-1.73)$ & Continuous $^{\mathrm{b}}$ & $1.48(1.28-1.72)$ & $1.20(1.00-1.43)$ \\
\hline
\end{tabular}

${ }^{a}$ Full Model: adjusted for age, sex, clinical indication for chest CT, image quality, and type of medical centre

${ }^{\mathrm{b}}$ Hazard ratios for continuous TAC/CAC scores are calculated per 1 standard deviation increase in CAC $(\mathrm{SD}=3.022)$ or TAC $(\mathrm{SD}=1.952)$

${ }^{\mathrm{c}}$ Non-cardiac events were fatal and non-fatal cases of stroke, aortic aneurysm/dissection and peripheral arterial occlusive disease

whereas people with severe $\mathrm{CAC}$ showed an increase in risk by a factor of 2.3. No statistically significant interaction was found between section thickness and TAC $(P=0.46)$ or CAC $(P=$ $0.89)$ and the use of intravenous contrast and TAC $(P=0.40)$ or CAC $(P=0.81)$ in relation to the risk of CVD events.

Table 5 shows the risk of stroke associated with increasing amounts of calcium in each of the three anatomically distinct parts of the thoracic aorta. Remarkably, strongest associations (and the only to reach statistical significance) were demonstrated for calcifications in the ascending aorta. Moderate calcification of the ascending aorta increased the risk of stroke by a factor 3.0 compared with no calcification, whereas moderate calcification of the descending aorta or supra-aortic branches increased the risk by a factor of 1.8 and 1.4 , respectively.

We also calculated multivariate-adjusted models with TAC and CAC sum scores entered simultaneously. Both scores were independently associated with the risk of (1) any CVD event increasing the risk by $25 \%(95 \% \mathrm{CI}, 13-38 \%)$ per $1 \mathrm{SD}$ increase in CAC score and 30\% (95\% CI, $17-44 \%)$ per $1 \mathrm{SD}$ increase in TAC score, respectively; and (2) coronary events increasing the risk by $42 \%(95 \% \mathrm{CI}, 25-61 \%)$ per $1 \mathrm{SD}$ increase in CAC score and 19\% (95\% CI, 4-36\%) per $1 \mathrm{SD}$ increase in TAC score, respectively. Only TAC was independently associated with the risk of non-cardiac events $(45 \%$ increased risk per $1 \mathrm{SD}$ increase in TAC score).

\section{Discussion}

We examined whether unrequested information from routine diagnostic chest $\mathrm{CT}$ in a clinical care population could be used as predictor of future disease. Severe coronary (CAC) or aortic (TAC) calcification-based on visual grading of calcified plaques - increased the risk of any CVD event almost 4-fold and 3-fold, respectively, after a mean follow-up of close to one and a half years. CAC was found to be a stronger predictor of coronary events, whereas TAC was more strongly associated with non-cardiac events.

More than 60 million diagnostic $\mathrm{CT}$ are performed annually in the USA and this number will likely increase as the population continues to age [15]. These diagnostic images 
Table 5 Risk of stroke $(n=58)$ associated with calcium in three anatomically distinct parts of the thoracic aorta

\begin{tabular}{|c|c|c|}
\hline \multirow[t]{2}{*}{ Site } & \multicolumn{2}{|c|}{ Hazard ratios $(95 \% \mathrm{CI})$} \\
\hline & Crude & Full model ${ }^{\mathrm{a}}$ \\
\hline \multicolumn{3}{|c|}{ Ascending aorta (0-3) } \\
\hline None $(0)$ & 1.0 & 1.0 \\
\hline Mild (1) & $3.2(1.9-5.6)$ & $2.2(1.3-4.0)$ \\
\hline Moderate (2) & $4.7(2.0-11.3)$ & $3.0(1.1-7.6)$ \\
\hline Severe (3) & $\operatorname{Inf}^{\mathrm{b}}$ & $\operatorname{Inf}^{\mathrm{b}}$ \\
\hline \multicolumn{3}{|c|}{ Descending aorta $(0-3)$} \\
\hline None $(0)$ & 1.0 & 1.0 \\
\hline Mild (1) & $3.0(1.6-5.8)$ & $1.8(0.9-3.7)$ \\
\hline Moderate (2) & $3.9(1.8-8.2)$ & $1.8(0.8-4.2)$ \\
\hline Severe (3) & $5.9(2.5-13.8)$ & $3.0(1.1-7.8)$ \\
\hline \multicolumn{3}{|c|}{ Supra-aortic branches $(0-2)$} \\
\hline None $(0)$ & 1.0 & 1.0 \\
\hline Moderate (1) & $2.4(1.2-4.5)$ & $1.4(0.7-2.7)$ \\
\hline Severe (2) & $3.1(1.7-5.9)$ & $1.7(0.8-3.4)$ \\
\hline
\end{tabular}

${ }^{a}$ Full Model: adjusted for age, sex, clinical indication for chest CT, image quality and type of medical centre

${ }^{b}$ Due to the limited number of stroke events, no cases occurred in the highest TAC category for the ascending aorta and consequently no hazard ratio could be calculated

contain unrequested, subclinical findings that could contain important prognostic information. Obtaining this information is 'free', as it is contained in routine care and comes at no additional exposure to ionising radiation. Currently, no data are available on the natural course and prognosis of these findings. The prognostic value (or lack thereof) of unrequested subclinical findings is important to indicate new ways of extracting clinically relevant information. Results from this study suggest that $\mathrm{CT}$ images obtained as part of routine clinical care do indeed convey important ancillary information.

We investigated the prognostic value of coronary and aortic calcifications as these are highly prevalent [6] and long recognised as prognostic markers of CVD in asymptomatic screening populations [1-5]. The prevalence of any CAC in our study (67\%) is comparable with these previous studies specifically aimed at screening for CAC and showing ranges between 53 and $69 \%$ [2, 16, 17]. Like these screening studies we show that $\mathrm{CAC}$ is a strong predictor of future CVD events. However, to our knowledge, our results are the first to demonstrate this association in a routine clinical care population. Furthermore, we used a simple visual grading to score calcifications on a heterogeneous set of CT protocols. Existing calcium scoring typically involves non-contrastenhanced, ECG-gated CT protocols with semi-automatic calcium scoring software. The scoring method we used consists of simple definitions, is reproducible, can be performed with a minimum of additional reviewing time and can be applied to a whole range of different CT protocols (including non-gated CT). Prognostic information on CVD risk can be appended to the CT report. Together with all relevant clinical data available from a patient's medical record, the clinician can decide whether additional work-up is required. This will result in a more consistent and clinically useful way of reporting unrequested subclinical findings.

Cardiovascular disease is one of the leading causes of death and hospitalisations in western society [18]. Major advances in knowledge about CVD risk factors have been made in recent decades and international guidelines for the primary prevention of CVD have been actively developed [19-21]. However, for a variety of reasons substantial groups of people at intermediate or high risk of CVD events according to existing guidelines are currently not recognised and treated as such $[22,23]$. This has prompted expert panels to state that the search for new strategies to detect patients who would benefit most from intensive primary prevention efforts is a clinically important objective [24, 25]. The present study is an attempt at defining such a new strategy.

Unfortunately, no information from patients' medical records was available for this study. This might have influenced our results in two ways. First, exclusion of patients with symptomatic CVD could now only be performed based on information from $\mathrm{CT}$ reports, possibly resulting in a number of symptomatic CVD patients being missed. This would have caused an overestimation of our risk ratios. However, we excluded approximately $16 \%$ of patients from the baseline clinical care cohort based on information from CT reports $(2,303 / 14,366)$ which is slightly more than the prevalence of symptomatic CVD $(11.2 \%)$ in the general white population of the USA [26]. Second, lack of information from medical records prevented us from adjusting our risk estimates for traditional CVD risk factors (hypertension, hyperlipidaemia, diabetes, smoking). Based on previous reports we expect that only limited attenuation of our age- and sex-adjusted risk estimates will occur when adjusting additionally for other CVD risk factors [3].

\section{Conclusion}

Unrequested subclinical findings - such as coronary and aortic calcifications - detected on routine care, diagnostic chest $\mathrm{CT}$ in a clinical care population can be used as a prognostic marker of future CVD events. This may be achieved with a simple visual scoring method that is easy to use in daily clinical practice. This offers a novel approach in trying to employ potentially valuable prognostic information contained in routine diagnostic imaging and may be used to identify patients who could benefit from primary preventive efforts. 
Acknowledgements The study was conducted by the PROVIDI study group. The PROVIDI Study Group consists of: J. Laméris (Dept. of Radiology, Academic Medical Center, Amsterdam), C. van Kuijk (Dept. of Radiology, Free University Medical Center, Amsterdam), W. ten Hove (Dept. of Radiology, Gelre Hospitals, Apeldoorn), M. Oudkerk (Dept. of Radiology, University Medical Center Groningen), A. Oen (Dept. of Radiology, Elkerliek Hospital, Helmond), J. Wildberger (Dept. of Radiology, Academic Hospital Maastricht), J. van Heesewijk (Dept. of Radiology, St. Antonius Hospital, Nieuwegein), W. Mali (Dept. of Radiology, University Medical Center Utrecht) and Y. van der Graaf (Julius Center for Health Sciences and Primary Care, University Medical Center Utrecht).

We acknowledge the help with data collection and administrative support by Cees Haaring (UMCU), Karin Flobbe (AZM), Mirjam
Borg (VUMC), Pascal Verzijl (Antonius), Jan Wolfers (AMC), Peter van Ooijen (UMCG), Willie Donkers (Elkerliek) and Robert Dragt (Gelre). This study was funded by a program grant from the Netherlands Organization for Scientific Research-Medical Sciences (NWO-MW) grant 40-00812-98-07-005). The funders had no involvement in the study design, data collection, analysis and interpretation, the writing of the report, or the decision to submit the paper for publication.

Open Access This article is distributed under the terms of the Creative Commons Attribution Noncommercial License which permits any noncommercial use, distribution, and reproduction in any medium, provided the original author(s) and source are credited.

\section{Appendix}

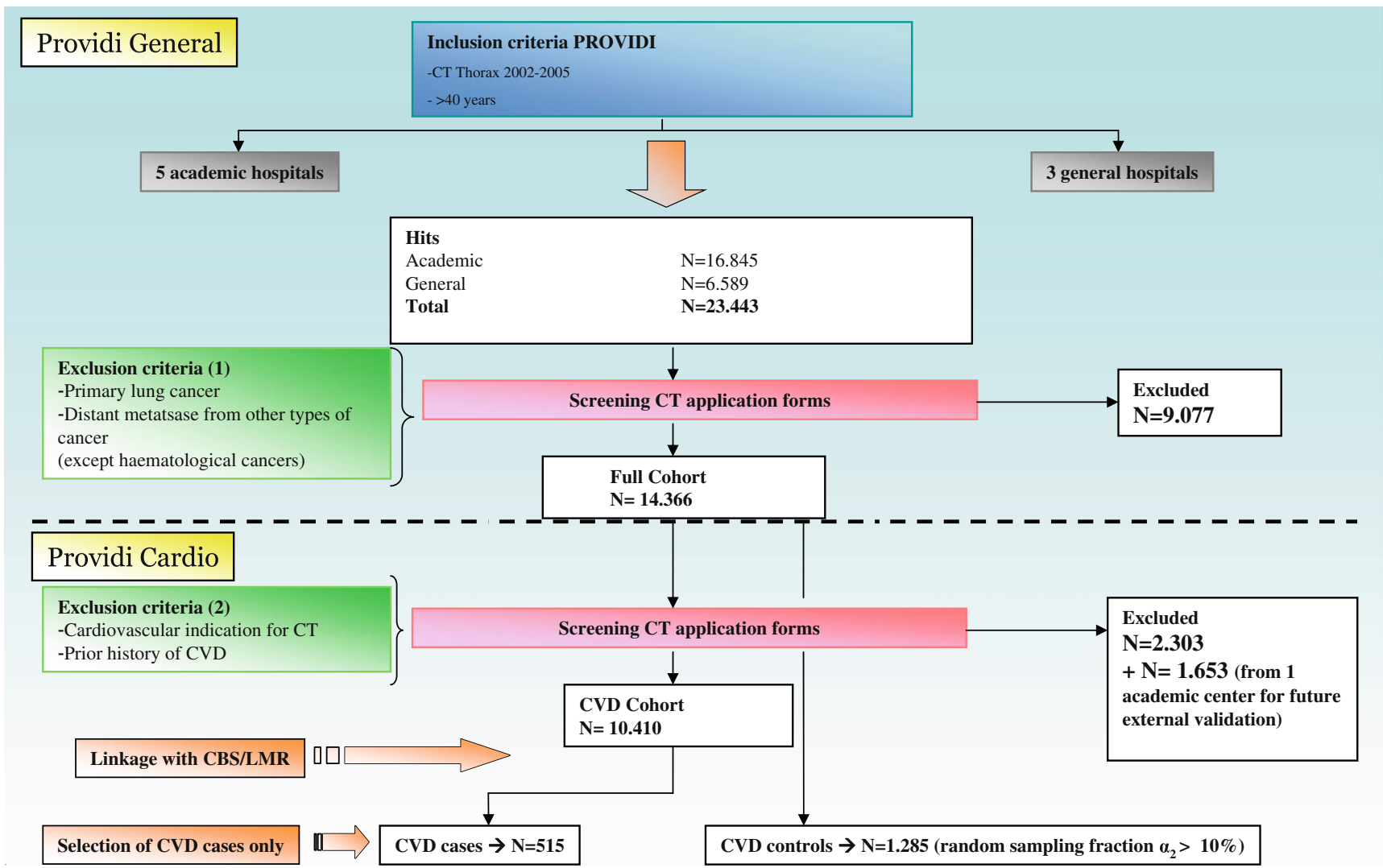

\section{References}

1. Arad Y, Goodman KJ, Roth M, Newstein D, Guerci AD (2005) Coronary calcification, coronary disease risk factors, C-reactive protein, and atherosclerotic cardiovascular disease events: the St. Francis Heart Study. J Am Coll Cardiol 46:158-165

2. Kondos GT, Hoff JA, Sevrukov A et al (2003) Electron-beam tomography coronary artery calcium and cardiac events: a 37-month follow-up of 5635 initially asymptomatic low- to intermediate-risk adults. Circulation 107:2571-2576

3. LaMonte MJ, FitzGerald SJ, Church TS et al (2005) Coronary artery calcium score and coronary heart disease events in a large cohort of asymptomatic men and women. Am J Epidemiol 162:421-429

4. O'Malley PG, Taylor AJ, Jackson JL, Doherty TM, Detrano RC (2000) Prognostic value of coronary electron-beam computed tomography for coronary heart disease events in asymptomatic populations. Am J Cardiol 85:945-948

5. Shaw LJ, Raggi P, Schisterman E, Berman DS, Callister TQ (2003) Prognostic value of cardiac risk factors and coronary 
artery calcium screening for all-cause mortality. Radiology 228:826-833

6. Odink AE, van der Lugt A, Hofman A et al (2007) Association between calcification in the coronary arteries, aortic arch and carotid arteries: the Rotterdam study. Atherosclerosis 193:408-413

7. Prentice RL (1986) A case-cohort design for epidemiologic cohort studies and disease prevention trials. Biometrika 73:1-11

8. Shemesh J, Henschke CI, Farooqi A et al (2006) Frequency of coronary artery calcification on low-dose computed tomography screening for lung cancer. Clin Imaging 30:181-185

9. De Bruin A, Kardaun JW, Gast A, Bruin E, van Sijl M, Verweij G (2004) Record linkage of hospital discharge register with population register: experiences at Statistics Netherlands. Stat J UN Econ Comm Eur 21:23-32

10. Herings RM, Bakker A, Stricker BH, Nap G (1992) Pharmacomorbidity linkage: a feasibility study comparing morbidity in two pharmacy based exposure cohorts. J Epidemiol Community Health 46:136-140

11. Paas GR, Veenhuizen KC (2002) Research on the validity of the LMR. Utrecht, Prismant

12. Reitsma JB, Kardaun JW, Gevers E, de Bruin A, van der Wal J, Bonsel GJ (2003) Possibilities for anonymous follow-up studies of patients in Dutch national medical registrations using the municipal population register: a pilot study. Ned Tijdschr Geneeskd 47:2286-2290

13. WHO International Classification of Diseases (ICD). Available via www.who.int/whosis/icd10/. Accessed 19 Sept 2009

14. Mackenbach JP, Van Duyne WM, Kelson MC (1987) Certification and coding of two underlying causes of death in The Netherlands and other countries of the European community. J Epidemiol Community Health 41:156-160

15. Brenner DJ, Hall EJ (2007) Computed tomography-an increasing source of radiation exposure. N Engl J Med 357:2277-2284

16. Budoff MJ, Shaw LJ, Liu ST et al (2007) Long-term prognosis associated with coronary calcification: observations from a registry of 25,253 patients. J Am Coll Cardiol 49:1860-1870
17. Wong N, Gransar H, Shaw LJ et al (2009) Thoracic aortic calcium versus coronary artery calcium for the prediction of coronary heart disease and cardiovascular disease events. JACC Cardiovasc Imaging 2:319-326

18. Murray CJ, Lopez AD (1997) Global mortality, disability, and the contribution of risk factors: global burden of disease study. Lancet 349:1436-1442

19. Assmann G, Cullen P, Schulte H (2002) Simple scoring scheme for calculating the risk of acute coronary events based on the 10-year follow-up of the prospective cardiovascular munster (PROCAM) study. Circulation 105:310-315

20. Conroy RM, Pyorala K, Fitzgerald AP et al (2003) Estimation of ten-year risk of fatal cardiovascular disease in Europe: the SCORE project. Eur Heart J 24:987-1003

21. Wilson PW, D'Agostino RB, Levy D, Belanger AM, Silbershatz H, Kannel WB (1998) Prediction of coronary heart disease using risk factor categories. Circulation 97:1837-1847

22. Greenland P, LaBree L, Azen SP, Doherty TM, Detrano RC (2004) Coronary artery calcium score combined with Framingham score for risk prediction in asymptomatic individuals. JAMA 291:210-215

23. Ridker PM, Paynter NP, Rifai N, Gaziano JM, Cook NR (2008) C-reactive protein and parental history improve global cardiovascular risk prediction: the Reynolds risk score for men. Circulation 118:2243-2251

24. Grundy SM, Cleeman JI, Merz CN et al (2004) Implications of recent clinical trials for the national cholesterol education program adult treatment panel III guidelines. Circulation 110:227-239

25. Naghavi M, Libby P, Falk E et al (2003) From vulnerable plaque to vulnerable patient: a call for new definitions and risk assessment strategies: part II. Circulation 108:1772-1778

26. Lloyd-Jones D, Adams R, Carnethon M et al (2009) Heart disease and stroke statistics -2009 update: a report from the American heart association statistics committee and stroke statistics subcommittee. Circulation 119:480-486 